\title{
Conocimiento y práctica del uso de equipos de protección personal en el contexto covid 19 en el personal de salud del servicio de UCI del Hospital El Carmen Huancayo 2020
}

Knowledge and practice of the use of personal protective equipment in the context of covid 19 in the health personnel of the ICU service of the Hospital EI Carmen Huancayo 2020

\author{
Anahi Indira De La Calle Castro ${ }^{1, a}$, Rebeca Tello Carhuanca ${ }^{1, b}$, Teresa Leonor Villegas Félix ${ }^{1, c}$, Edith \\ Suasnabar Cueva ${ }^{1, d}$
}

\section{RESUMEN}

Objetivo: Determinar la relación entre el conocimiento y la práctica del uso de equipos de protección personal en el contexto COVID 19 en el personal de salud del servicio de UCI del hospital el Carmen Huancayo 2020. El área de estudio corresponde a las Ciencias médicas y de salud. Material y métodos: El método de esta investigación será el científico que consiste en un método de investigación usado principalmente. El diseño de investigación es correlacional, no experimental y de corte transversal, el tipo de investigación, de acuerdo a la finalidad realizada será básica, el nivel de Investigación será descriptivo - correlacional. Resultados: Los resultados muestran que el $56 \%$ del personal de UCI del Hospital El Carmen tiene un conocimiento medio del uso de EPP y el 68\% cumple medianamente con el uso de EPP en el contexto Covid - 19. Conclusiones: Concluyendo que existe relación significativa entre el nivel de conocimiento y la aplicación de las medidas de del personal de UCI del Hospital El Carmen, 2020. ( $\mathrm{p}$ valor $=0,001<0,05)$.

PALABRAS CLAVE: Conocimiento, práctica, uso de equipos de protección personal.

\section{SUMMARY}

Objective: To determine the relationship between knowledge and practice of the use of personal protective equipment in the context of COVID 19 in the health personnel of the ICU service of the hospital el Carmen Huancayo 2020. The area of study corresponds to medical and health sciences. Material and methods: The method of this research will be scientific which consists of a research method mainly used. The research design is correlational, nonexperimental and cross-sectional, the type of research, according to the purpose will be basic, the level of research will be descriptive - correlational. Results: The results show that $56 \%$ of the ICU personnel of Hospital El Carmen have an average knowledge of the use of PPE and 68\% comply moderately with the use of PPE in the Covid - 19 context. Conclusion: We conclude that there is a significant relationship between the level of knowledge and the application of the measures of the ICU personnel of Hospital El Carmen,2020. ( $\mathrm{p}$ value $=0.001<0.05$ ).

KEY WORDS: Knowledge, practice, use of personal protective equipment.

\footnotetext{
Universidad Privada de Huancayo Franklin Roosevelt. Huancayo, Perú.

Docente; Licenciada en Enfermería; Magister; ORCID ID: 0000-0003-1206-1321

Docente; Licenciada en Enfermería; Magister; ORCID ID: 0000-0003-3915-4405

Docente; Licenciada en Enfermería; Magister; ORCID ID: 0000-0001-8385-1596

Docente; Licenciada en Enfermería; ORCID ID: 0000-0002-2202-8720
} 


\section{INTRODUCCIÓN}

A nivel mundial un informe de la Pan American Health Organization (PAHO) menciona que a fines del pasado año en el mes de diciembre es identificado un nuevo coronavirus o SARS-CoV-2 que es el agente etiológico de neumonía (COVID-19) en la China ciudad de Wuhan, Hay incertidumbre en relación a la historia natural de la enfermedad, así como de los mecanismos de transmisión del virus y su diseminación del virus. En el mes de febrero del 2020, en China se publicó un estudio con 72314 casos registrados, de los cuales $44672(62 \%)$ se diagnosticaron como SARS$\mathrm{CoV}-2$ (1).

Para el uso de los equipos de protección personal (EPP) la Organización Mundial de la Salud (OMS) dio a conocer recomendaciones tanto en los centros sanitarios como en los domicilios, así como durante la manipulación de mercancías. Además, se analizan las interrupciones actuales que sufre la cadena mundial de suministro y se tratan aspectos que cabe tener en cuenta para tomar decisiones durante periodos de escasez grave de estos equipos. en vista de ello sugiere la OMS aplicar estrategias para mejorar su disponibilidad. Por ejemplo, conviene reducir hasta lo indispensable la necesidad de EPP en los entornos de atención sanitaria, asegurarse de que los EPP se utilizan de forma racional y correcta y coordinar los mecanismos de gestión de la cadena de suministro ( figura 1) (2).

En el Perú, se aplican las normas internacionales, sin embargo, en el mes de marzo del 2020 se buscaron protocolos de manejo, guías de práctica clínica, y documentos técnicos que aborden los procedimientos a seguir para el uso de EPP por el personal de salud asistencial ante casos sospechosos, probables $\mathrm{o}$ confirmados de COVID19 (3).

En el Hospital El Carmen el personal ha seguido las normas nacionales para el uso de Equipos de Protección Personal para hacerlas llegar al personal de salud, pero es necesario conocer cuánto de su uso conocen cuánto es usado por el personal de la Unidad de Cuidados Intensivos, el área de mayor riesgo.

En el contexto de esta nueva enfermedad conocida como COVID 1, con su agente etiológico SARS$\mathrm{CoV}-2$, el uso de equipos de protección personal es una herramienta eficaz para la protección del personal de salud que se encuentra en la primera línea de atención y manejo de pacientes críticos en las Unidades de Cuidados Intensivos. Conocedores de la alta demanda de pacientes que requieren el ingreso a las unidades de cuidados intensivos generando el incremento de riesgo de contagio de médicos, enfermeras y técnico de enfermería y según el reporte de colegios profesionales a la fecha hay una gran cantidad de personal contagiado $\mathrm{y}$ un número considerable de defunciones.

Un antecedente de estudio es el de la Organización Mundial de la Salud, en la investigación Uso racional del equipo de protección personal frente a la COVID-19 y aspectos que considerar en situaciones de escasez graves, 2020 (4).

Donde los resultados y conclusiones principales fueron que al momento de considerar si se adoptan los métodos descritos, el manejo de las mascarillas durante el procedimiento de descontaminación es un paso crucial, debiéndose evitar una manipulación excesiva. Además, deben existir sistemas para inspeccionar meticulosamente las mascarillas antes de cada ciclo de reacondicionamiento a fin de comprobar la integridad y el mantenimiento de la forma; si las mascarillas están dañadas o no son aptas para su reutilización, deben desecharse inmediatamente. Los principales aspectos que deben tenerse en cuenta para considerar aceptable un método de reacondicionamiento son: 1) eficacia del método para desinfectar/esterilizar el equipo; 2) el mantenimiento de la eficacia filtrante de la mascarilla; 3 ) la conservación de la forma de la mascarilla y por ende de su ajuste; y 4) la seguridad para el usuario de la mascarilla (por ejemplo, toxicidad después del reacondicionamiento). Los siguientes métodos deben evitarse porque dañan las mascarillas o provocan toxicidad o pérdida de eficacia filtrante: lavado, esterilización por vapor de agua a $134{ }^{\circ} \mathrm{C}$, desinfección con lejía/hipoclorito sódico o alcohol, e irradiación en hornos de microondas. Los hornos de microondas han demostrado cierto efecto biocida cuando se combinan con humedad gracias al efecto combinado de la radiación y el vapor de agua caliente; sin embargo, algunos problemas que deben tenerse en cuenta son: i) la falta de un examen sustancial de las capacidades de radiación de los hornos de microondas convencionales en relación con la desinfección de mascarillas auto filtrantes, ii) la imposibilidad de garantizar una distribución uniforme del vapor de agua y iii) el riesgo de que la banda nasal metálica pueda entrar en combustión. El uso de radiación ultravioleta podría ser una alternativa, aunque la baja capacidad de penetración de la luz ultravioleta puede hacer que esta no llegue al material interno de las mascarillas auto filtrantes o no pueda atravesar los plisados o pliegues. Otro antecedente es el del Ministerio de Salud del Perú, 
en la investigación: Documento técnico: Prevención y atención de personas afectadas por COVID 19 en el Perú; Lima, Perú. 2020 (5).

Donde los resultados y conclusiones principales fueron que la información generada en todos los establecimientos de salud públicos y privados que realizan actividades de prevención y control del COVID-19 es consolidada por la Oficina de Estadística en sus diferentes niveles. El Registro de Información Operacional del COVID 19 en sus diferentes niveles utilizará un aplicativo web específicamente diseñado, además del sistema HIS MINSA. Es responsabilidad del Ministerio de Salud y demás entidades del Sector Salud en el nivel nacional, regional o local, programar, adquirir, almacenar y distribuir los productos farmacéuticos, dispositivos médicos y productos sanitarios para el abastecimiento de la red asistencial de salud pública a nivel nacional. El Ministerio de Salud, las DIRIS/DISA/DIRESA/ GERESA, o quien haga sus veces en los pliegos correspondientes, financian a través de las diferentes fuentes de financiamiento (Recursos ordinarios y recursos directamente recaudados), las actividades de prevención, detección, diagnóstico, control y tratamiento del COVID-19. Dicho financiamiento debe garantizarse por los pliegos correspondientes. Para el caso de los asegurados al Seguro Integral de Salud (SIS), el financiamiento de las prestaciones relacionadas a la prevención, diagnóstico, control y tratamiento del COVID-19 será de acuerdo a la normatividad vigente y a los mecanismos de pago establecidos por el Seguro Integral de Salud. Debido al corto tiempo de evolución de la pandemia evidencias científicas limitadas, la información contenida en el presente Documento Técnico se encuentra sujeta a modificaciones en tanto exista nueva información a nivel internacional disponible. Se recomienda revisar de forma permanente la página web oficial del Ministerio de Salud para acceder a las publicaciones oficiales más recientes.

El objetivo del estudio fue determinar la relación entre el conocimiento y la práctica del uso de equipos de protección personal en el contexto COVID 19 en el personal de salud del servicio de UCI del hospital el Carmen Huancayo.

\section{MATERIAL Y MÉTODOS}

El método de investigación fue el científico, usado principalmente en la producción de conocimiento en las ciencias. Para ser llamado científico, un método de investigación debe basarse en la empírica y en la medición, sujeto a los principios específicos de las pruebas de razonamiento (5).

El tipo de Investigación por su finalidad fue básico, porque mejoro el conocimiento y comprensión de los fenómenos sociales, así mismo fue fundamento de toda investigación (6).

El nivel de esta investigación fue descriptivo correlacional, porque intentó establecer la relación existente entre dos o más variables, pero sin pretender dar una explicación completa del fenómeno investigado (7).

El diseño de la investigación será correlacional, no experimental y transversal correlacional, porque tuvo como propósito medir el grado de relación que exista entre dos o más variables, en un contexto en particular. Y transversal porque se midió la variable una vez en el tiempo (7).

No experimental porque no se manipuló las variables De hecho, no hay condiciones o estímulos a los cuales se expongan los sujetos del estudio. Los sujetos son observados en su ambiente natural, en su realidad (6).

Esquemáticamente es expresada de esta forma:

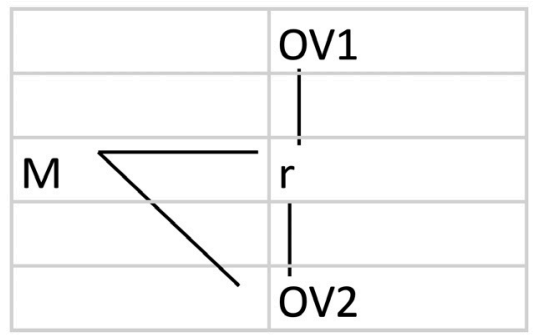

\section{Dónde:}

$\mathrm{M}=$ Muestra:

OV1 = Observación de la variable 1: Conocimiento de equipos de protección personal en el contexto COVID 19

OV2 = Observación de la Variable 2: Práctica del uso de equipos de protección personal en el contexto COVID 19

$\mathrm{r}=$ Correlación entre la variable 1 y la variable 2

La población estuvo conformada por el personal de UCI, conformado como sigue: 


\begin{tabular}{cc}
\hline PERSONAL & TOTAL \\
\hline Enfermeras & 15 \\
Técnicas & 10 \\
TOTAL & 25 \\
\hline
\end{tabular}

Se realizó un muestreo probabilístico, es decir a criterio de las investigadoras la muestra se ha determinado que sea censal es decir el total de la población.

\section{Técnicas e instrumentos de recolección de datos}

La técnica de recopilación de datos es la encuesta que utiliza un conjunto de procedimientos estandarizados de investigación mediante los cuales se recoge y analiza una serie de datos de una muestra de casos representativa de una población o universo más amplio, del que se pretende explorar (6).

El instrumento a usar es el cuestionario, que es utilizado para recolectar datos, que consiste en un conjunto de preguntas respecto a una o más variables a medir, teniendo en cuenta los problemas de investigación (6).

En esta investigación se tuvo 2 cuestionarios: El primer cuestionario de 18 ítems para conocimiento de uso de EPP en el contexto Covid-19, y el segundo instrumento fue una lista de cotejo de 11 ítems para Práctico de uso de EPP en el contexto Covid-19.

De acuerdo a Rosas y Zúñiga un cuestionario es confiable para un valor de alfa de cronbach $\geq 0,05$ (7).

Para el cuestionario de Conocimiento de uso de EPP en el contexto Covid-19 el valor de alfa de cronbach es de 0,861 lo que indica que el instrumento es aplicable el valor fue:

\begin{tabular}{cc}
\hline \multicolumn{2}{c}{ Estadísticas de fiabilidad } \\
\hline Alfa de Cronbach & $\mathrm{N}$ de elementos \\
0,803 & 18 \\
\hline
\end{tabular}

El valor de 0,803 de alfa de Cronbach indica que el instrumento es confiable.

Para el cuestionario de Práctica de uso de EPP en el contexto Covid-19 el valor de alfa de cronbach es de 0,861 lo que indica que el instrumento es aplicable el valor fue:

\begin{tabular}{cc}
\hline \multicolumn{2}{c}{ Estadísticas de fiabilidad } \\
\hline Alfa de Cronbach & $\mathrm{N}$ de elementos \\
0,814 & 11 \\
\hline
\end{tabular}

El valor de 0,814 de alfa de Cronbach indica que el instrumento es confiable

\section{RESULTADOS}

\section{Resultado Descriptivo}

Resultado de Variable 1: Conocimiento de uso de EPP en el contexto Covid-19

En la tabla 1 y el gráfico 1 observamos que no se tiene conocimiento del uso de EPP en el contexto Covid - 19 el 32\%, tiene conocimiento medio el 56\% y conoce el $12 \%$. Por lo tanto, el $56 \%$ del personal de UCI del Hospital El Carmen tiene un conocimiento medio del uso de EPP en el contexto Covid - 19

Tabla 1. Conocimiento de uso de EPP en el contexto Covid-19.

\begin{tabular}{ccc}
\hline Nivel & Frecuencia & Porcentaje \\
\hline No conoce & 8 & $32 \%$ \\
$\begin{array}{c}\text { Conocimiento } \\
\text { medio }\end{array}$ & 14 & $56 \%$ \\
Conoce & 3 & $12 \%$ \\
Total & 25 & $100 \%$ \\
\hline
\end{tabular}

\section{CONOCIMIENTO DE USO DE EPP CONTEXTO COVID-19}

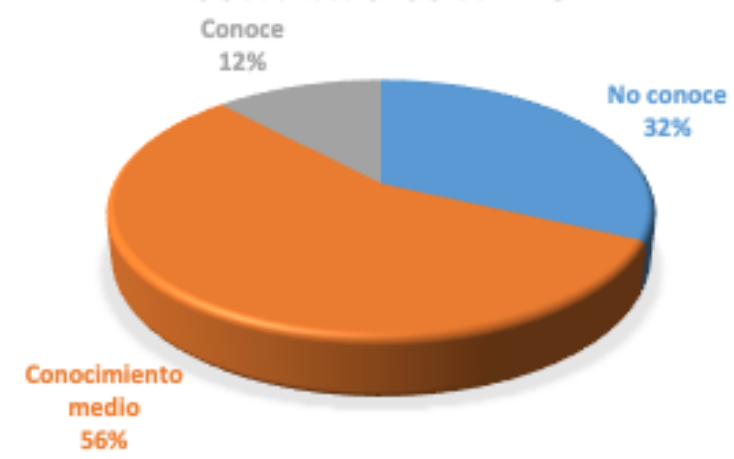

Gráfico 1. Conocimiento de uso de EPP en el contexto Covid-19 
Tabla 2. Práctica de uso de EPP en el contexto Covid-19

\begin{tabular}{lcr}
\hline Nivel & Frecuencia & Porcentaje \\
\hline No cumple & 4 & $16 \%$ \\
Cumple medianamente & 17 & $68 \%$ \\
Cumple & 4 & $16 \%$ \\
Total & 25 & $100 \%$ \\
\hline
\end{tabular}

Fuente: Lista de cotejo de práctica de uso de EPP en el contexto Covid-19

Resultado de Variable 2: Conocimiento de uso de EPP en el contexto Covid-19

En la tabla 2 y el gráfico 2 observamos que no cumple con el uso de EPP en el contexto Covid - 19 el $16 \%$, cumple medianamente el $68 \%$ y cumple el $16 \%$. Por lo tanto, el 68\% del personal de UCI del Hospital El Carmen cumple medianamente con el uso de EPP en el contexto Covid - 19.

\section{PRACTICA DE USO DE EPPP CONTEXTO COVID-19}

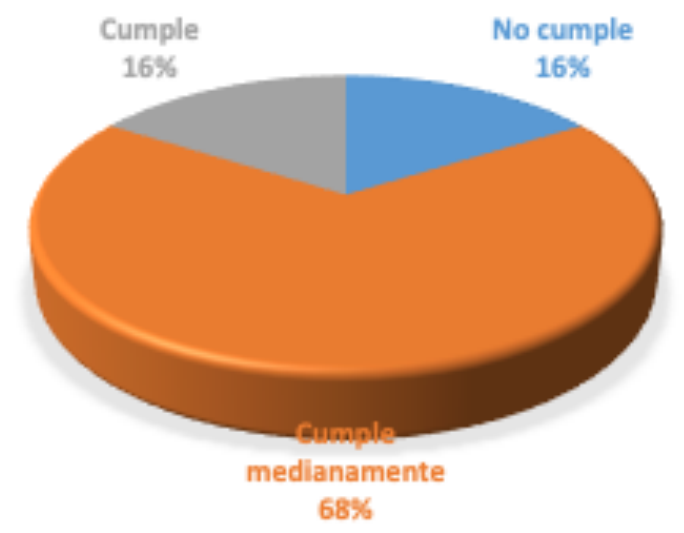

Gráfico 2. Práctica de uso de EPP en el contexto Covid-19

\section{Prueba de Hipótesis}

$\mathrm{H} 0=$ No existe relación significativa entre el conocimiento y la práctica del uso de equipos de protección personal en el contexto COVID 19 en el personal de salud del Servicio de UCI del hospital el Carmen Huancayo 2020.

$\mathrm{HI}=$ Existe relación significativa entre el conocimiento y la práctica del uso de equipos de protección personal en el contexto COVID 19 en el personal de salud del servicio de UCI del hospital el Carmen Huancayo. Nivel de significancia: $\alpha=0,05$.

El valor de Rho de Spearman es de 0,611 lo que nos indica que la correlación es directa y moderada y la significancia bilateral ( $p$ valor $=0,001<0,05)$ (tabla $3)$.

\section{Conclusión Estadística}

Por lo tanto, se rechaza la hipótesis nula y se acepta la de investigación en el sentido que existe relación significativa entre el nivel de conocimiento y la aplicación de las medidas de bioseguridad en profesionales de enfermería del Hospital Domingo Olavegoya - Jauja,2018. (p valor $=0,001<0,05$ ).

\section{DISCUSIÓN}

Los resultados del objetivo general nos muestran que existe relación significativa entre el nivel de conocimiento y la aplicación de las medidas de bioseguridad en profesionales de enfermería del Hospital Domingo Olavegoya - Jauja ,2018. (p valor $=0,001<0,05$ ). Donde, el $56 \%$ del personal de UCI del Hospital El Carmen tiene un conocimiento medio del uso de EPP en el contexto Covid - 19 y el $68 \%$ cumple medianamente con el uso de EPP en el contexto Covid - 19 .

Tabla 3. Rho de Spearman para conocimiento y aplicación de las medidas de bioseguridad.

\begin{tabular}{lllrr}
\hline & & VARIABLE 1 & VARIABLE 2 \\
\hline & & Coeficiente de correlación & 1,000 & $0,611^{* *}$ \\
\multirow{2}{*}{ Rho de Spearman } & & Sig. (bilateral) & & 0,001 \\
& & N & 25 & 25 \\
& & Coeficiente de correlación & $0,611^{* *}$ & 1,000 \\
& Práctica de uso de EPP & Sig. (bilateral) & 0,001 & \\
& & $\mathrm{~N}$ & 25 & 25 \\
\hline
\end{tabular}

**. La correlación es significativa en el nivel 0,01 (bilateral).

Fuente: Base de d SPSS 
En este sentido un estudio similar fue el la Organización Mundial de la Salud, en la investigación Uso racional del equipo de protección personal frente a la COVID-19 y aspectos que considerar en situaciones de escasez graves, 2020 (4). Donde los resultados y conclusiones principales fueron que al momento de considerar si se adoptan los métodos descritos, el manejo de las mascarillas durante el procedimiento de descontaminación es un paso crucial, debiéndose evitar una manipulación excesiva.

Y el del Ministerio de Salud del Perú, en la investigación Documento técnico. Prevención y atención de personas afectadas por COVID 19 en el Perú; Lima, Perú. 2020 (5). Donde los resultados y conclusiones principales fueron que la información generada en todos los establecimientos de salud públicos y privados que realizan actividades de prevención y control del COVID-19 es consolidada por la Oficina de Estadística en sus diferentes niveles. El Registro de Información Operacional del COVID 19 en sus diferentes niveles utilizará un aplicativo web específicamente diseñado, además del sistema HIS MINSA.

Por otro lado, teóricamente el Ministerio de Salud del Perú, refiere que el manejo de los casos sospechosos, probables o confirmados de COVID-19 implica el reconocimiento temprano de signos y síntomas de la enfermedad respiratoria aguda grave inusual, aislamiento del caso según prácticas de prevención y control de infecciones (PCI), monitoreo y terapia de soporte precoz, recolección de muestras para diagnóstico de laboratorio, manejo de fallo respiratorio (8).

\section{CONCLUSIONES}

Se ha determinado que existe relación significativa entre el nivel de conocimiento y la aplicación de las medidas de bioseguridad en profesionales de enfermería del Hospital Domingo Olavegoya - Jauja, $2018(\mathrm{p}$ valor $=0.001<0,05)$.

Se ha identificado que, el 56\% del personal de UCI del Hospital El Carmen tiene un conocimiento medio del uso de EPP en el contexto Covid - 19.

Se ha identificado que, el 68\% del personal de UCI del Hospital El Carmen cumple medianamente con el uso de EPP en el contexto Covid - 19 .

\section{Correspondencia:}

Edith Suasnabar Cueva

Correo electrónico: esuasnabar@uroosevelt.edu.pe

\section{REFERENCIAS BIBLIOGRAFICAS}

1. Pan American Health Organization. Especificaciones Técnicas de dispositivos médicos paa la gestión de casos COVID-19 en los servicios de salud. Washington DC: Pan American Health Organization; 2020. (Citado el 13 de diciembre del 2020) Disponible en: https://iris.paho.org/bitstream/ handle/10665.2/51980/OPSPHEIMCovid1920001 spa.pdf? sequence $=1 \&$ is Allowed $=\mathrm{y}$

2. Organización Mundial de la Salud. Uso racional del equipo de protección personal frente al COVID 19 y aspectos que considerar en situaciones de escasez graves. Ginebra: Organización Mundial de la Salud; 2020. (Citado el 13 de diciembre del 2020) Disponible en: https://apps.who.int/iris/ bitstream/handle/10665/331810/WHO-2019-nCoVIPC PPE use-2020.3-spa.pdf

3. EsSalud. Recomendaciones para el uso de equipo de protección personal (EPP) por el personal de salud asistencial ante casos sospechosos, probables o confirmados de COVID - 19. Lima, Perú: EsSalud; 2020. (Citado el 13 de diciembre del 2020) Disponible en: http://www.essalud.gob.pe/ietsi/ pdfs/guias/Recomendaciones_para_el_uso_de_EPP_ COVID_19.pdf

4. Organización mundial de la Salud. Uso racional del equipo de protección personal frente a la COVID-19y aspectos que considerar en situaciones de escasez graves. Ginebra: Organización Mundial de la Salud; 2020.

5. Ministerio de Salud. Documento técnico. Prevención y atención de personas afectadas por COVID 19 en el Perú. Lima, Perú: Ministerio de Salud; 2020.

6. Kerlinger FN. Enfoque conceptual de la investigación del comportamiento. Ciudad de México: Editorial Interamericana; 2002.

7. Rosas A, Zúñiga J . Estadística Descriptiva e Inferencial I. s.l. : Fascículo 3. Correlación y regresión lineales. Colegio de Bachilleres; 2010.

8. Ministerio de Salud. Alerta Epidemiológica: Código: AE-010-2020. Actualización de alerta epidemiológica ante el riesgo de introducción del COVID-19, en el Perú. Lima, Perú: Ministerio de Salud; 2020.

Recibido: $15 / 12 / 2020$

Aceptado: 14/04/2021 